\title{
A method for Verification of Treatment Delivery in HDR Prostate Brachytherapy using a Flat Panel Detector for both Imaging and Source
}

\section{Tracking}

Ryan L Smith ${ }^{1,2}$, Annette Haworth ${ }^{2,3}$, Vanessa Panettieri ${ }^{1}$, Jeremy L Millar ${ }^{1,2}$, and Rick D Franich ${ }^{1,2}$

${ }^{1}$ Alfred Health Radiation Oncology, The Alfred Hospital, Melbourne, 3004 VIC, Australia

${ }^{2}$ School of Science, RMIT University, Melbourne, 3000 VIC, Australia

${ }^{3}$ Physical Sciences, Peter MacCallum Cancer Centre, East Melbourne, 3000 VIC, Australia

KEY WORDS : Brachytherapy, treatment verification, HDR prostate brachytherapy, flat

panel detector

\begin{abstract}
Purpose: Verification of high dose rate (HDR) brachytherapy treatment delivery is an important step, but is generally difficult to achieve. A technique is required to monitor the treatment as it is delivered, allowing comparison with the treatment plan and error detection. In this work we demonstrate a method for monitoring the treatment as it is delivered and directly comparing the delivered treatment with the treatment plan in the clinical workspace. This treatment verification system is based on a flat panel detector (FPD) used for both pretreatment imaging and source tracking.

Methods: A phantom study was conducted to establish the resolution and precision of the system. A pre-treatment radiograph of a phantom containing brachytherapy catheters is acquired and registration between the measurement and treatment planning system (TPS) is performed using implanted fiducial markers. The measured catheter paths immediately prior to treatment were then compared with the plan. During treatment delivery, the position of the ${ }^{192} \mathrm{Ir}$ source is determined at each dwell position by measuring the exit radiation with the FPD, and directly compared to the planned source dwell positions.
\end{abstract}


Results: The registration between the two corresponding sets of fiducial markers in the TPS and radiograph yielded a registration error (residual) of $1.0 \mathrm{~mm}$. The measured catheter paths agreed with the planned catheter paths on average to within $0.5 \mathrm{~mm}$. The source positions measured with the FPD matched the planned source positions for all dwells on average within $0.6 \mathrm{~mm}$ (s.d. 0.3, min. 0.1, max. $1.4 \mathrm{~mm}$ ).

Conclusion: We have demonstrated a method for directly comparing the treatment plan with the delivered treatment that can be easily implemented in the clinical workspace. Pre-treatment imaging was performed, enabling visualisation of the implant before treatment delivery and identification of possible catheter displacement. Treatment delivery verification was performed by measuring the source position as each dwell was delivered. This approach using a FPD for imaging and source tracking provides a non-invasive method of acquiring extensive information for verification in HDR prostate brachytherapy. 


\section{Introduction}

40 Verification of high dose rate (HDR) brachytherapy treatment delivery is an important step, but is generally difficult to achieve. Poor execution of a planned treatment would have significant clinical impact, as high doses are delivered in seconds and mistakes in an individual fraction cannot be easily rectified. Two brachytherapy review articles ${ }^{1,2}$ highlight the need for an independent and comprehensive treatment verification system in HDR brachytherapy to ensure patient safety.

Many systems for field and dose verification are available and considered essential in external beam radiotherapy (EBRT) to ensure the treatment is delivered as prescribed ${ }^{3,4}$. In HDR brachytherapy, current treatment quality assurance processes are often limited to pre-treatment manual cross checking of delivery parameters, such as: correct patient plan selection, indexer length, source activity, total treatment time and correct transfer tube connection to the implant. In many cases, human errors are the main cause of treatment delivery mistakes ${ }^{5}$. An independent system to verify these aspects of HDR brachytherapy treatment delivery is required.

Approaches to treatment delivery verification in HDR brachytherapy have mainly employed in vivo dosimetry techniques ${ }^{6,7}$. The measured dose at one (or several) points within (or near) the treatment volume by a dosimeter, is then used as a surrogate for correct treatment delivery. One significant challenge with this approach is accurately knowing the relative positions of both the source and dosimeter to be able to interpret the measurement. An unexpected discrepancy in the dose relative to the expected value can only be interpreted as a detected delivery error if these positions are known. In practice, this can be difficult and a moderate spatial uncertainty can translate to a much higher dosimetric uncertainty in the high dose gradients typical in HDR brachytherapy.

An alternative approach for treatment verification is to determine the position of the source throughout the treatment delivery, identify where these source positions are relative to the catheters/applicator and where they occur in relation to the surrounding anatomy. From this information it is possible to verify the treatment was delivered as planned and in principle reconstruct the dose that was delivered to the tumour and/or organs at risk. In order to achieve routine treatment verification in HDR brachytherapy, the monitoring technique must allow the delivered treatment to be easily compared to the treatment plan in the clinical workspace. 
Approaches to measure the position of a HDR brachytherapy source have previously been reported $^{8-10}$, but these systems have usually been employed as pre-treatment, quality assurance devices and are not necessarily suitable for in vivo measurements to be used for treatment delivery verification.

75 We have previously reported on our approach to source position verification based on a flat panel detector (FPD) system that captures a $2 \mathrm{D}$ response of the ${ }^{192} \mathrm{Ir}$ radiation exiting the patient's body. In that work we showed that these two dimensional measurements could be analysed for source position, providing valuable information for the verification of correct delivery of treatment plans ${ }^{11}$.

80 In this work we demonstrate a method for directly comparing the delivered treatment with the planned treatment by: performing a registration between the two frames of reference, comparing the measured catheter paths immediately prior to treatment with the plan and then directly comparing the measured source dwell positions with the planned dwell positions. We describe in a phantom study the potential clinical implementation of our novel source tracking system for HDR prostate patient treatment verification. This approach, using a FPD embedded in the treatment couch, provides a non-invasive method of acquiring information for verification in HDR brachytherapy.

\section{Method}

The phantom (with known geometry) is utilized here to establish the resolution and precision of the system. The required steps detailed below are: (i) pre-treatment imaging of the implant using the same FPD as is used for tracking the source; (ii) registering the imaging/tracking coordinate system with the TPS space; (iii) comparison of the implant geometry with the plan; (iv) comparison of measured source dwell positions with the plan. This phantom based experiment represents a single end-to-end test of the system as a treatment verification process.

A rectangular phantom was constructed from 10 solid water slabs (Gammex RMI, Middleton, WI, USA) (each of $300 \times 300 \times 10 \mathrm{~mm}$ ), containing a simulated brachytherapy implant of 18 plastic catheters (6F Proguide, Elekta, Stockholm, Sweden) spaced at $10 \mathrm{~mm}$ intervals, in a grid-like design as depicted in figure 1. Also embedded in the phantom were three fiducial 
markers to represent prostate anatomical markers which are typically used for patient positioning in the external beam component of prostate radiotherapy treatment ${ }^{12}$.

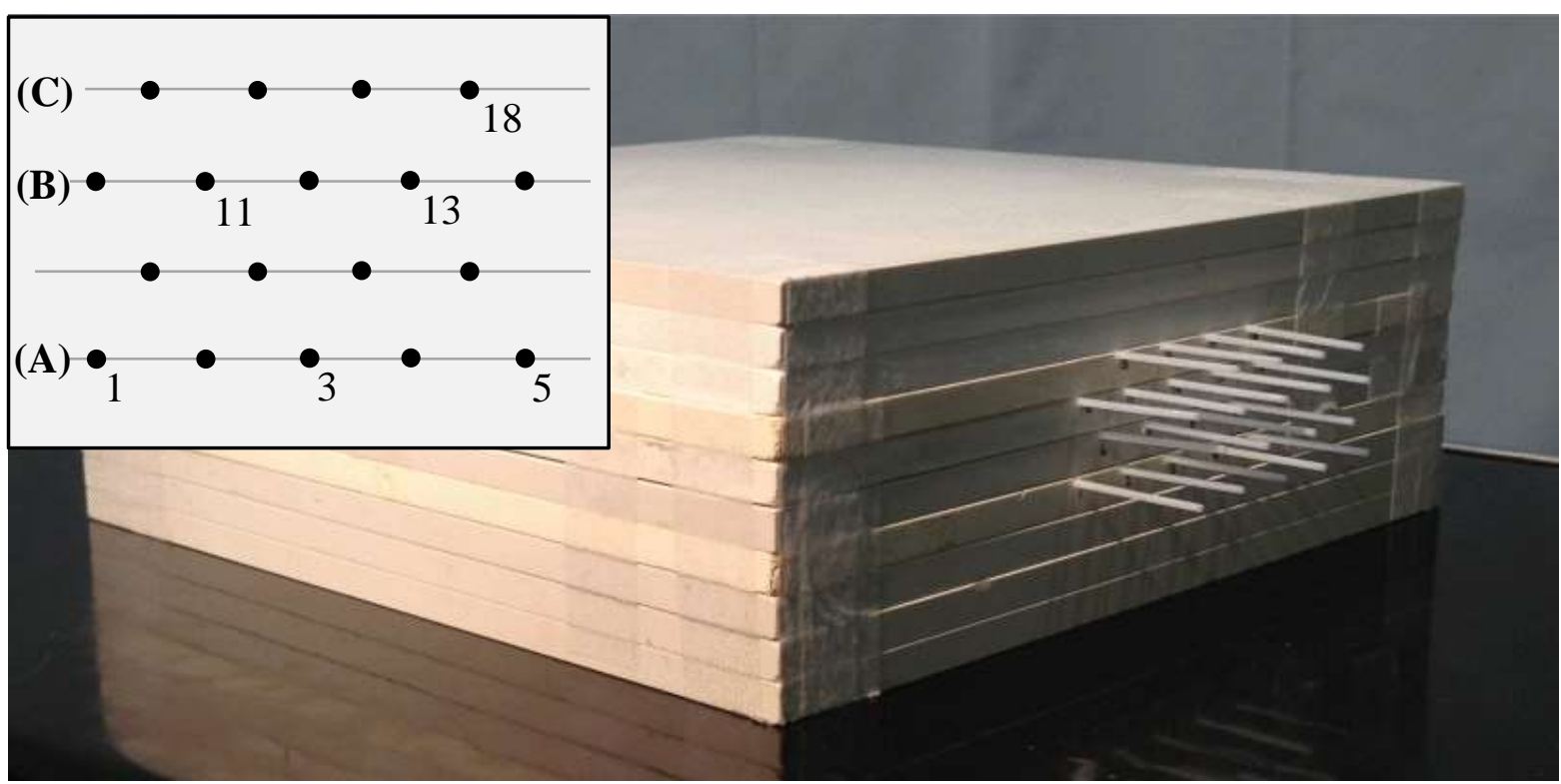

Figure 1. The solid water phantom used for this work constructed from solid water slabs, containing 18

'Proguide' plastic brachytherapy catheters, arranged in a grid like fashion to mimic the template grid used clinically. Three fiducial markers were also included in the phantom to represent anatomical markers implanted into the prostate tissue. The inset shows the catheter layout - the catheters used in the simulated plan are numbered.

110 The phantom was placed on the CT scanner couch, CT markers were inserted into all 18 catheters for visualisation in the scan, and the phantom scanned using our standard clinical HDR prostate brachytherapy protocol $(2.5 \mathrm{~mm}$ spaced, $2.5 \mathrm{~mm}$ thick slices). The CT image data was imported into the Oncentra brachytherapy treatment planning system (TPS) (Oncentra v4.3, Elekta, Stockholm, Sweden) and the 'implanted' catheters in the phantom reconstructed.

A treatment plan was created using a set of six selected catheters that covered the left to right and superior to inferior range of the simulated implant. The catheter layout is shown in the inset of figure 1, with the treatment plan consisting of three planes (A, B and C) with catheters containing planned dwell positions labelled (1, 3, 5, 11, 13 and 18). The three 'anatomical' fiducial markers were identified in the TPS by placing patient points at the centre of each. The plane representing the position of the imaging plane of the FPD below the couch was created in 
the TPS and all planned catheter points, dwell positions and fiducial marker points were projected on to this plane for direct comparison with the measured data.

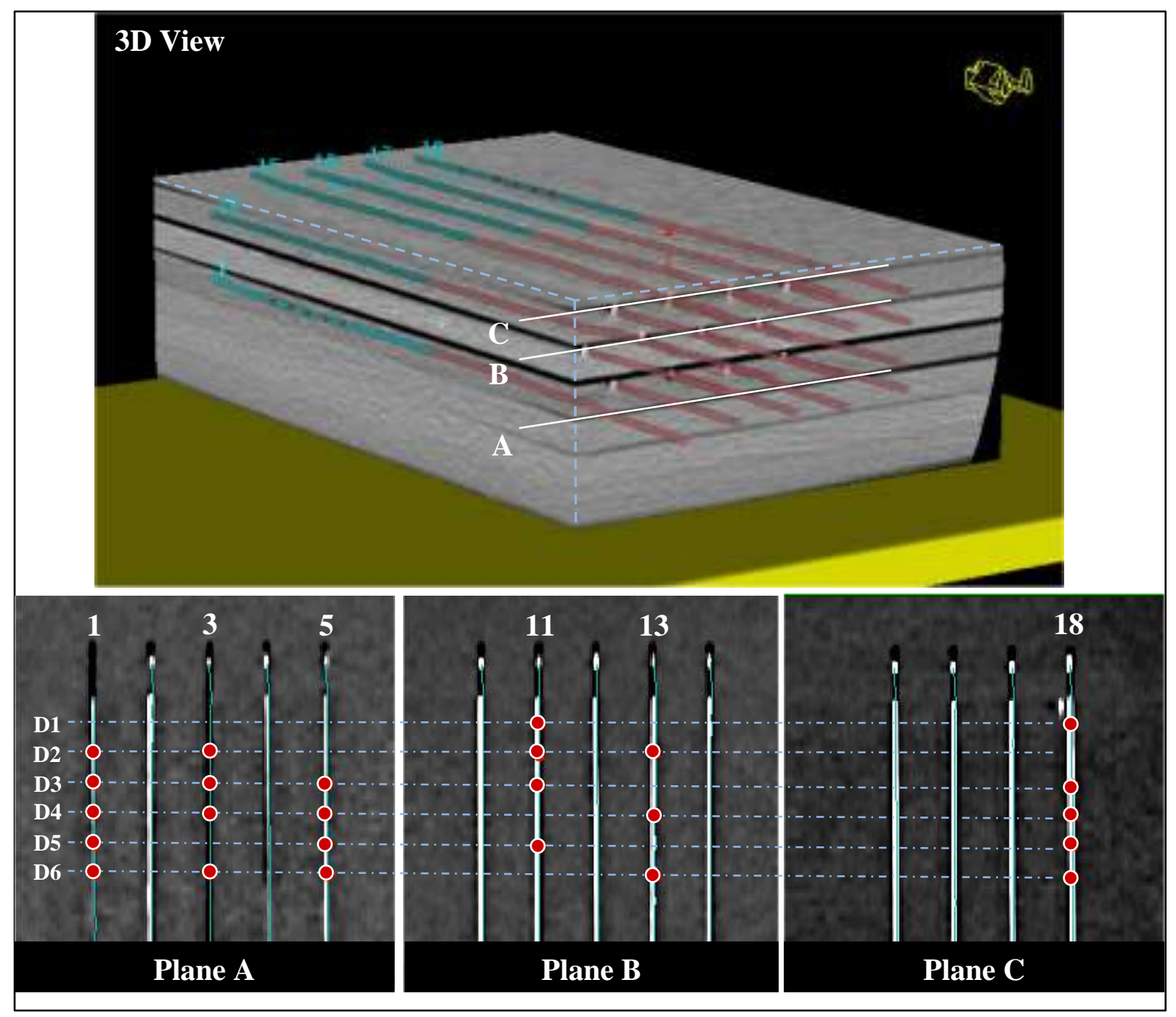

Figure 2. An illustration of the treatment plan showing the 3D transparent representation of the phantom. Three planes of catheters containing the planned dwell positions are shown.

The phantom was transferred to the brachytherapy treatment room, hosting the treatment afterloader (microSelectron, Elekta, Stockholm, Sweden), and set up on the treatment couch. The brachytherapy treatment couch includes a carbon fibre couch top with a flat panel detector (aS-500, Varian Medical Systems, Palo Alto, CA, USA) integrated into the couch assembly 
below (figure 3). Radio-opaque x-ray dwell position markers (different from those used at CT) with $10 \mathrm{~mm}$ spaced lead markers were inserted into the six selected catheters used in the treatment plan. An x-ray radiograph was acquired using the FPD as the image receptor and a kilovoltage x-ray source (QRad OTS x-ray suspension system, Carestream Health, Rochester, NY, USA) positioned at a known focus to image distance (FID) of $1000 \mathrm{~mm}$ above the phantom. A ball bearing (BB) array attached to the x-ray source was used during the capture of the radiograph in order to correct for image divergence when comparing radiograph coordinates to orthogonal projections from the TPS. The setup of the phantom on the treatment couch, the FPD position and the imaging geometry is illustrated in figure 3.

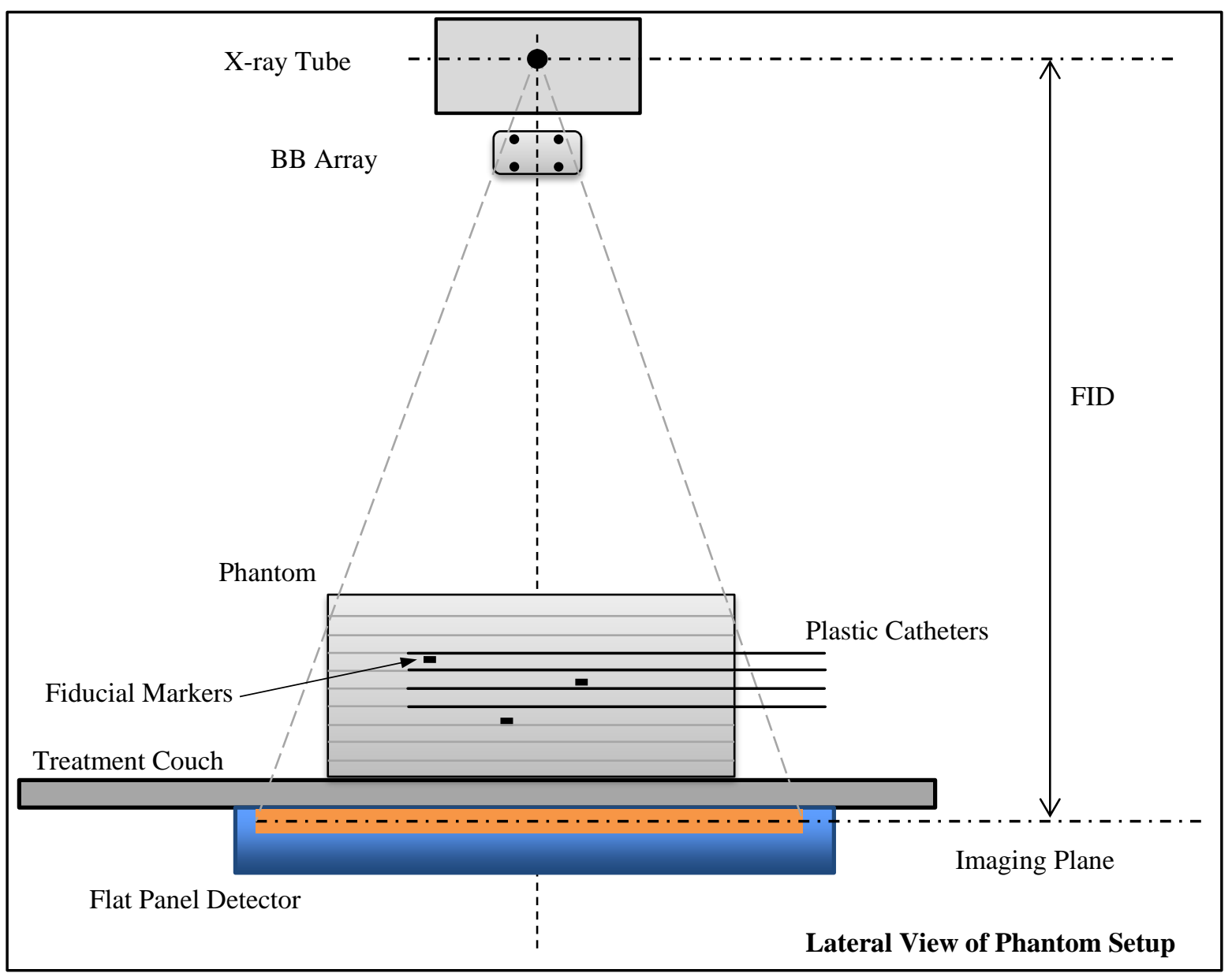

Figure 3. The setup of the solid water phantom on the treatment couch above the flat panel detector, with three fiducial markers depicted. A ball bearing (BB) array is included to correct for image divergence. The distance from the kilovoltage $\mathrm{x}$-ray source to the flat panel detector imaging plane, focus to imager distance (FID) is $1000 \mathrm{~mm}$. 
The x-ray dwell position markers were then removed from the catheters, the treatment afterloader transfer tubes connected and the treatment plan was delivered. At each source dwell position an acquisition was made with the FPD of the ${ }^{192}$ Ir source exit radiation. The captured distribution at each source dwell was processed using shape analysis and the source position in the imaging plane of the FPD determined. An example of the distribution from the ${ }^{192}$ Ir source captured by the FPD is shown in figure 5(a) and the procedure for performing shape analysis on this distribution is described in Smith et al ${ }^{11}$.

\section{Registration of Planned (TPS) with Measured (FPD) space}

To achieve verification of the treatment delivery by direct comparison with the treatment plan, a relationship between the planned (TPS) space and the measured (FPD) space was established.

160 The positions of the three fiducial markers embedded in the solid water phantom were determined in the acquired radiograph. A divergence correction was applied to the measured fiducial marker positions using a correction factor determined by the BB array. These measured fiducial marker positions were paired with the corresponding three fiducial markers previously identified in the treatment plan. A non-iterative, least squares method of registration was performed ${ }^{13}$ in $2 \mathrm{D}$ to determine the desired rigid spatial transformation (rotation and translation) between the planned and measured spaces. The reported registration error from this process is the residual or the sum of the squared differences for each registered marker pair. The registration process is illustrated in figure 4(a) showing the registration between the two corresponding sets of fiducial markers in the TPS and FPD space and the resulting transformation matrix, $\mathrm{T}$, which establishes a link between the two frames of reference.

The phantom setup on the treatment couch mimics that at CT, with the base of the phantom flat on the couch, in order to exclude tilt and roll effects. But in the clinical case, position deviations are expected. In order mimic potential patient setup differences between CT and treatment imaging, the phantom was re-positioned to incorporate tilt (rotation of the phantom in the superior-inferior direction) and roll (rotation of the phantom in the left-right direction). A radiograph was acquired with the phantom tilted 2 degrees in the superior direction. The phantom was then positioned to have a roll of 2 degrees and another radiograph was acquired. For each case, the fiducial markers were identified in the radiograph and a registration performed with the TPS using the method previously described. The registration quality was 
determined for each case in order to assess the impact of patient setup position on the fiducial marker registration.

\section{Catheter Path Comparison - "Pre-Treatment Imaging”}

The aim of pre-treatment imaging is to evaluate the position of the implanted catheters and determine any geometric changes that may have occurred between treatment planning and immediately prior to treatment delivery. Using the spatial transformation established in the previous section, the planned catheter paths can be directly compared with the catheter paths identified in the acquired radiograph.

The path of each catheter in the radiograph was identified by manually appending the centres of the $10 \mathrm{~mm}$ spaced x-ray dwell position markers, using in-house software. The planned catheter paths from the TPS were transformed to the measurement (FPD) frame of reference, using the transformation established by the fiducial marker registration, as illustrated in figure 4(b). The corresponding planned and measured catheter positions were compared by determining the absolute difference in the plane of the FPD. Each catheter tip position was compared as well as each of the marker points along the length of the catheter, providing a measure of stable implant geometry between planning and pre-treatment. While no implant geometry changes are expected in this phantom study, this step will have significance in the patient case. In addition, the level of agreement between measured source dwell positions and planned positions cannot be greater than that permitted by any changes in implant geometry. This must be taken into account when setting tolerance limits for error detection. 


\section{(a) Registration between Plan (TPS space) and Measurement (FPD space)}

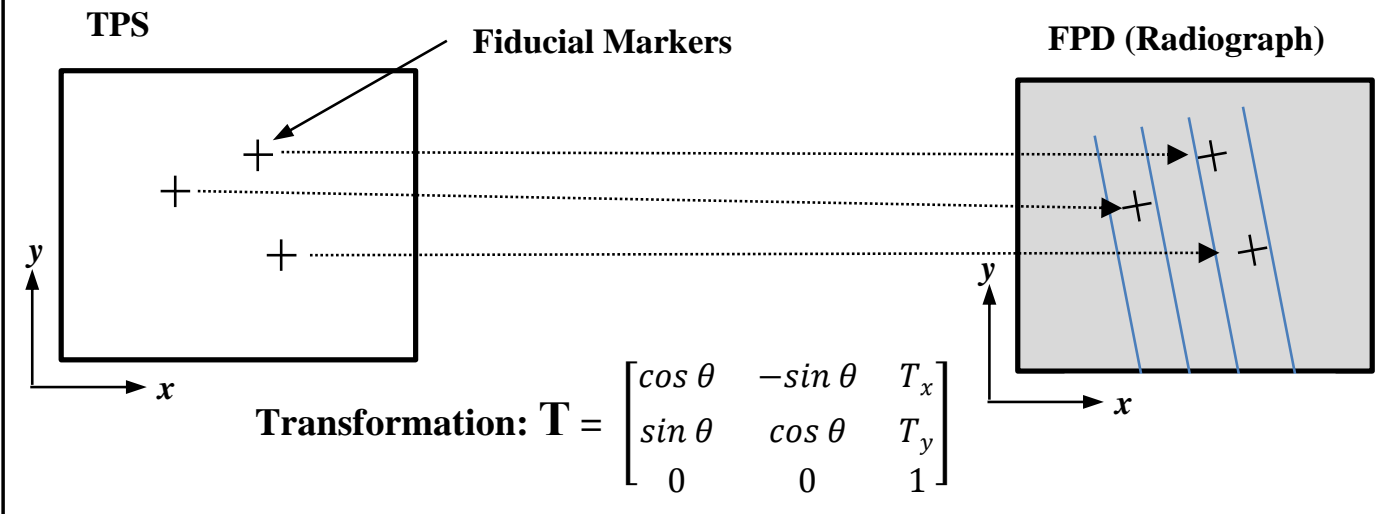

\section{(b) Transform the Planned Catheter Paths}

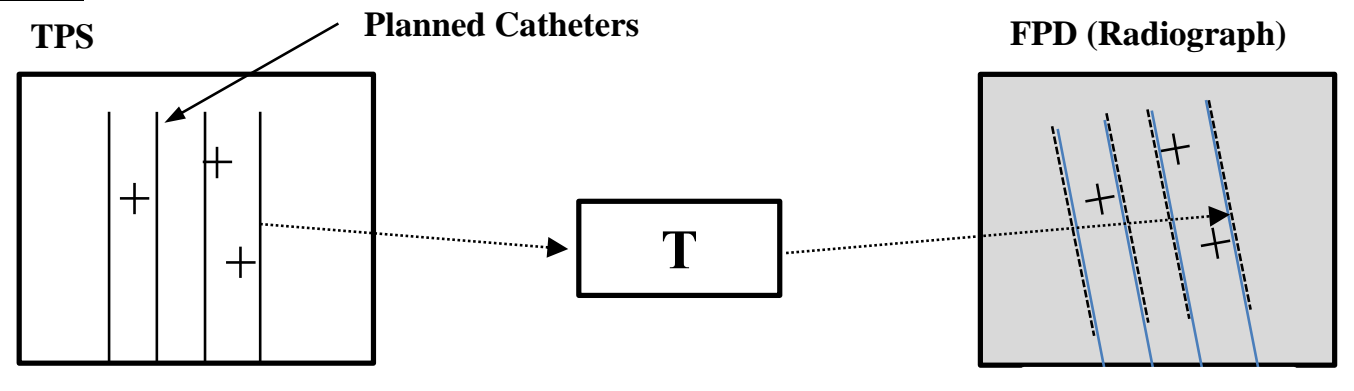

\section{(c) Transform the Planned Dwell Positions}

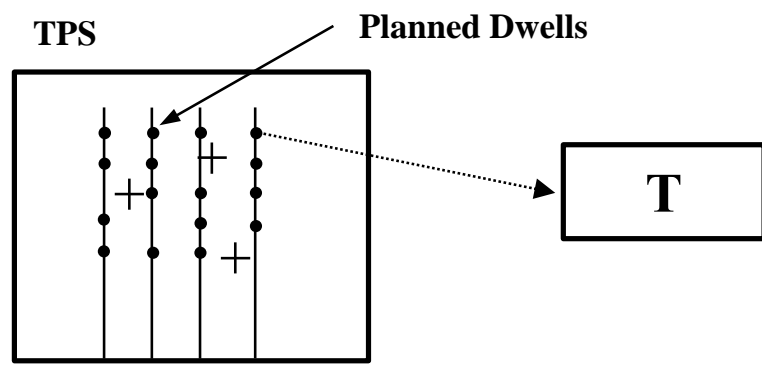

FPD (Radiograph)

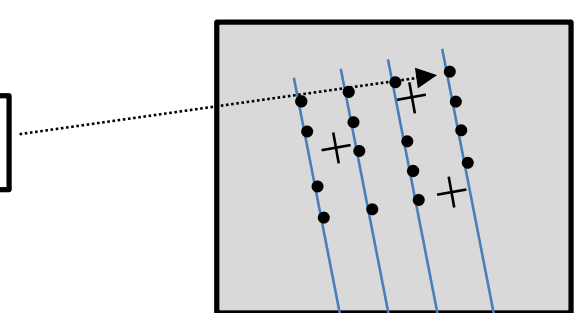

Figure 4. (a) An illustration showing the 2D registration process for corresponding fiducial marker

points in the treatment plan and the measured radiograph. The resulting transformation matrix, $T$, where $\theta$ represents the counter-clockwise rotation of the fiducial markers about the origin, and $T_{x}$ and $T_{y}$ represent a translation in the $\mathrm{x}$ and $\mathrm{y}$ directions respectively. (b) The transformation of planned catheter paths into the measurement frame of reference for direct comparison with catheter paths immediately prior to treatment. (c) The transformation of planned dwell positions for direct comparison with measured dwell positions acquired with the FPD. 


\section{Dwell Position Comparison - "Source Tracking”}

215 The source dwell positions from the treatment plan were also transformed to the FPD frame of reference ready for comparison with the measured dwell positions, as illustrated in figure 4(c). The measured dwell positions, determined from the FPD captured distribution of ${ }^{192}$ Ir exit radiation, were then compared to each corresponding planned dwell position.

\section{Independent Verification Measurement}

The shape analysis of the captured distribution of exit radiation provides a measurement of source position in the plane of the FPD. An independent method to validate this measured source position method was performed by acquiring a dual exposure radiograph during the source dwell. That is, while the FPD was capturing a distribution from the ${ }^{192} \operatorname{Ir}$ source (Figure 5(a)), the x-ray tube was then pulsed to expose the FPD to a simultaneous projection image (Figure 5(b)). The subtraction of the source dwell image from the dual exposure image then showed the position of the physical source capsule (Figure 5(c)), which was used as an independent validation of source position. This dual capture process is used strictly as an independent validation of the source position measured in this phantom study, and is not proposed to be performed during clinical implementation of this treatment verification method.
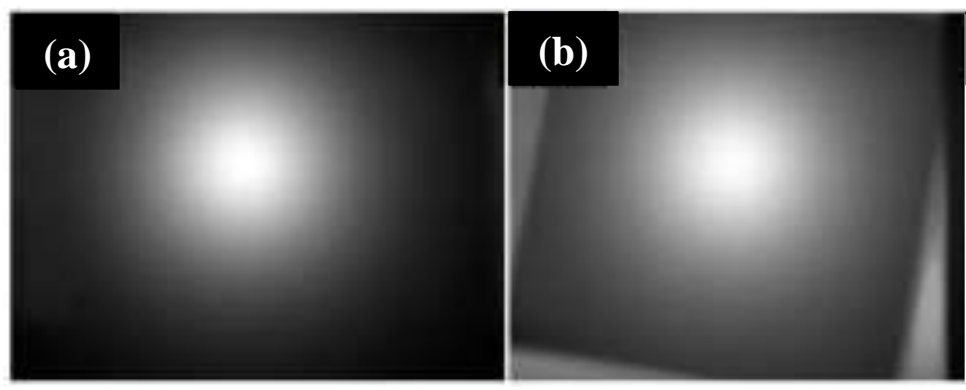

\section{(c)}

Source Cable and Capsule

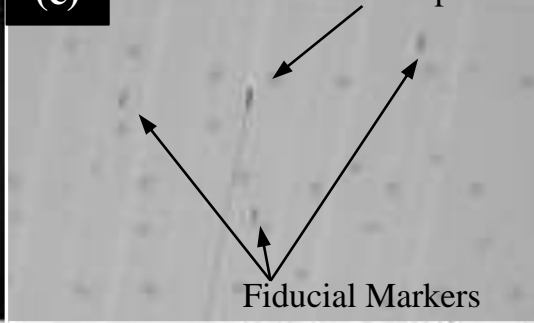

Figure 5. (a) The captured exposure of the ${ }^{192} \mathrm{Ir}$ source using the FPD. (b) The simultaneous FPD exposure of the ${ }^{192} \mathrm{Ir}$ source and the projection image of the phantom. (c) The result of subtraction of (a) from (b), magnified view to show the physical source capsule used for independent position 
verification. The source cable, voids from the other empty catheters and fiducial markers are also visible. 


\section{Results}

Registration of Planned (TPS) with Measured (FPD) space

The $2 \mathrm{D}$ rigid registration between the two corresponding sets of fiducial markers yielded a registration error (residual) of $1.0 \mathrm{~mm}$. The registered set of fiducial markers can be seen in figure 6(a), with the planned markers (black crosses) obscuring the measured markers (white crosses) as the two sets of markers are virtually coincident, indicating the high level of agreement following registration. The mean absolute difference calculated between each corresponding fiducial marker is $0.6 \mathrm{~mm}$, which is comparable to the pixel dimension of the radiograph image of $0.8 \mathrm{~mm}$ (PortalVision ${ }^{\mathrm{TM}}$ Reference Manual, Varian Medical Systems, Palo Alto, CA). The paths of the six catheters used for the treatment plan and the $10 \mathrm{~mm}$ spaced $\mathrm{x}-$ ray dwell position markers can also be seen in figure 6(a).

The registration residual for the phantom rolled by 2 degrees was $1.2 \mathrm{~mm}$ and for the phantom tilted by 2 degrees was $1.1 \mathrm{~mm}$. The 2 degree rotation in each orientation produced a registration quality that was not significantly different from the flat phantom case.

\section{Catheter Path Comparison - "Pre-Treatment Imaging"}

255 To evaluate the planned and measured catheter tip position agreement, the TPS catheter tip position was compared to the catheter tip identified in the radiograph. The absolute agreement for all six catheter tips was on average $0.4 \mathrm{~mm}$. This absolute measure does not reflect the direction of possible catheter movement along the longitudinal axis and so the relative tip difference along the long axis of the catheter is shown in table 1.

To evaluate possible overall change in the catheter position, the measured $\mathrm{x}$-ray dwell position marker points were compared to the TPS catheter marker points for each catheter over a length of $100 \mathrm{~mm}$ from the catheter tip, as illustrated in figure 6(b). A summary of these corresponding catheter points is shown in table 2. 
Table 1. The difference between the TPS catheter tip position and the catheter tip position identified on the radiograph along the axis of the catheter path.

\begin{tabular}{lccc|cc|c}
\hline \hline Catheter No. & $\mathbf{1}$ & $\mathbf{3}$ & $\mathbf{5}$ & $\mathbf{1 1}$ & $\mathbf{1 3}$ & $\mathbf{1 8}$ \\
\hline Tip Difference (mm) & 0.2 & 0.2 & -0.1 & -1.0 & -0.6 & 0.2 \\
\hline \hline
\end{tabular}

270

Table 2. The absolute difference between the TPS catheter points and the appended $x$-ray dwell position marker points on the radiograph at $10 \mathrm{~mm}$ intervals along the catheter path. This comparison evaluates the overall catheter path deviation between planning and treatment delivery.

\begin{tabular}{lccc|cc|c}
\hline \hline Catheter No. & $\mathbf{1}$ & $\mathbf{3}$ & $\mathbf{5}$ & $\mathbf{1 1}$ & $\mathbf{1 3}$ & $\mathbf{1 8}$ \\
\hline & & & & & & \\
Mean (mm) & 0.4 & 0.4 & 0.3 & 0.7 & 0.7 & 0.5 \\
Minimum (mm) & 0.2 & 0.2 & 0.0 & 0.4 & 0.1 & 0.2 \\
Maximum (mm) & 0.7 & 0.7 & 0.6 & 1.1 & 0.9 & 0.9 \\
\hline \hline
\end{tabular}

\section{Dwell Position Comparison - "Source Tracking"}

The planned source dwell positions were transformed on to the radiograph and are shown as

solid red circles in figure 6(c). The measured source dwell positions were determined at all dwells in the treatment plan and are shown overlayed on the planned dwell positions in figure 6(d) as black diagonal crosses. The planned and measured dwells all agree on average within $0.6 \mathrm{~mm}$ (s.d. 0.3, min. 0.1, max. $1.4 \mathrm{~mm}$ ) and a summary of agreement is shown in table 3 for all dwell positions. The differences shown are the vector lengths - there were no systematic directional trends. 


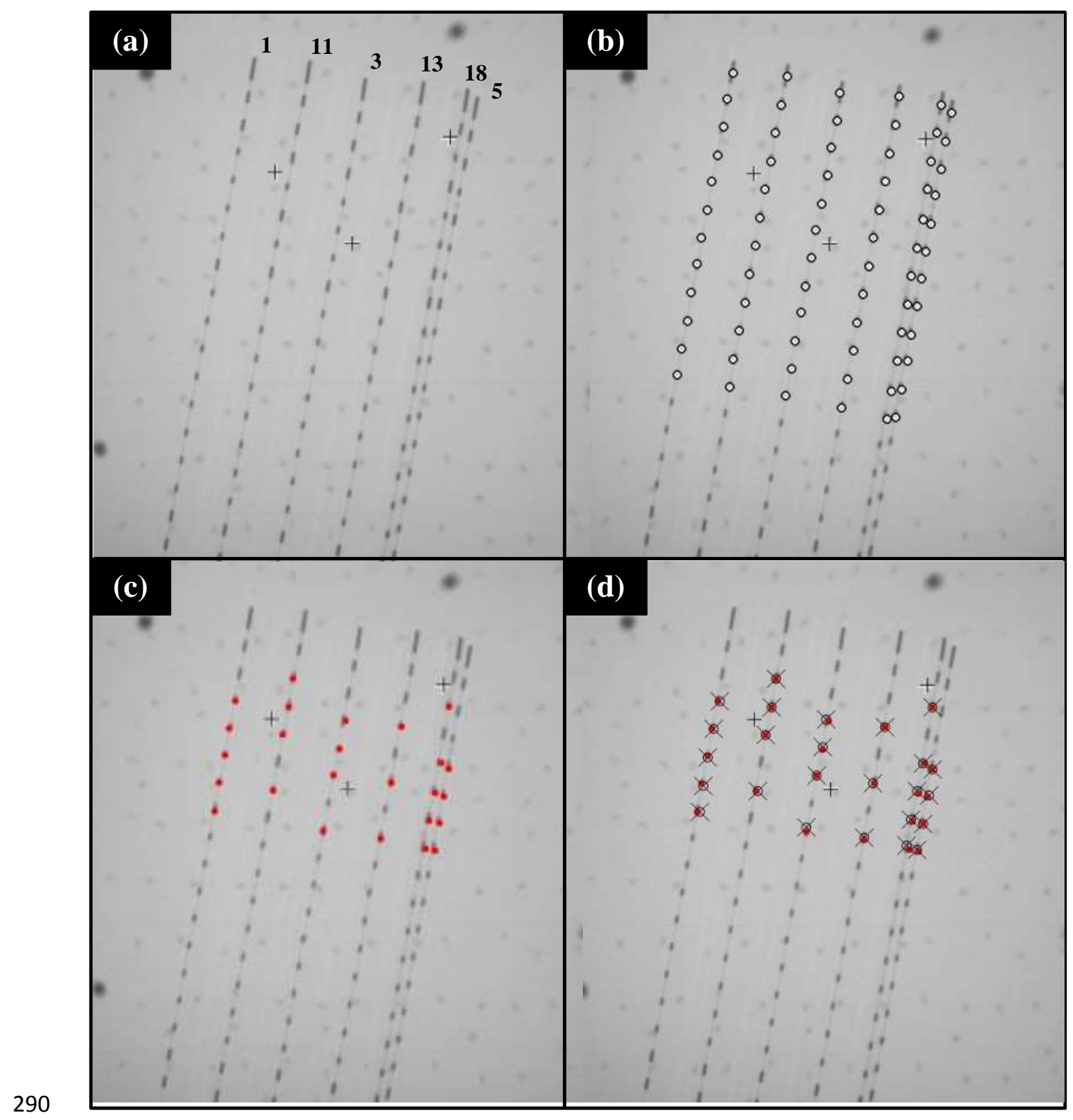

Figure 6. (a) The radiograph of the phantom on the treatment couch showing the x-ray dwell position markers in each labelled catheter and the appended fiducial markers (+). (b) The planned catheter markers transformed into the measurement frame of reference and displayed on the radiograph (०). (c) The red solid circles represent the dwell positions from the treatment plan displayed on the radiograph. 
(d) The black diagonal cross and circle show the measured dwell positions displayed with the planned dwell positions for all six catheters included in the treatment plan.

Table 3. The absolute difference between measured and planned positions for each dwell position in the treatment plan. The dwell positions are defined in figure 2, where D1 is toward the tip of the catheter (according to the Oncentra TPS convention). The distance from the detector plane to the catheter containing the ${ }^{192} \mathrm{Ir}$ source, 'source to detector distance' (SDD) for each catheter is also shown.

\begin{tabular}{lccc|cc|c}
\hline \hline Catheter No. & $\mathbf{1}$ & $\mathbf{3}$ & $\mathbf{5}$ & $\mathbf{1 1}$ & $\mathbf{1 3}$ & $\mathbf{1 8}$ \\
\hline SDD (mm) & 80 & 80 & 80 & 100 & 100 & 110 \\
\hline Difference (mm) & & & & & \\
D1 & 0.5 & 0.5 & & 0.6 & & 0.6 \\
D2 & 0.6 & 0.4 & 0.3 & 0.4 & & 0.3 \\
D3 & 0.7 & 0.5 & 0.2 & & 0.7 & 0.7 \\
D4 & 0.8 & & 0.4 & 0.8 & & 1.0 \\
D5 & 0.8 & 0.9 & 0.8 & & 0.7 & 1.4 \\
D6 & 0.9 & & & & \\
\hline \hline
\end{tabular}

The measured position of the centre of the source capsule obtained from the image subtraction method (figure 5(c)) was used to verify the measured source position determined by the shape analysis procedure. All source positions agreed on average by $0.8 \mathrm{~mm}$ (s.d. 0.3, Min. 0.2, Max $1.4 \mathrm{~mm}$ ) the agreement at $95 \%$ confidence interval of $0.7 \mathrm{~mm}$ for all dwells (data not shown). 


\section{Discussion}

Registration of Planned (TPS) with Measured (FPD) space

Geometric registration between the TPS frame of reference and the measurement frame of reference was required in order to perform absolute position comparisons. We have achieved the registration step using corresponding 'anatomical' fiducial markers, creating a geometric transformation between the TPS and the FPD space. This permitted the TPS plan parameters to be mapped onto the FPD for direct comparison with the measured values, including catheter position and source dwell positions.

The quality of geometric registration will influence the level of agreement observed in these direct comparisons. A measure of the registration quality is the registration residual and for this phantom study, the residual was $1.0 \mathrm{~mm}$. The relative position between the fiducial markers is static between CT (for treatment planning) and pre-treatment imaging, therefore the principal factor influencing the registration quality is uncertainty in identifying the fiducial markers in the radiograph image.

Accurately appending the fiducial marker positions on the radiograph image is subject to the pixel dimension. The size of the pixels in the radiograph is $0.8 \mathrm{~mm}$ square $^{14}$ and so due to the uncertainty of selecting exactly the centre of the fiducial marker (partial volume), we define the uncertainty as \pm 1 pixel $(0.8 \mathrm{~mm})$. This uncertainty value is consistent with the absolute mean difference between corresponding registered markers $(0.6 \mathrm{~mm})$, suggesting that a deviation of $0.8 \mathrm{~mm}$ for radiograph marker identification does not significantly influence the quality of registration.

For the clinical case, additional factors will influence the registration quality, such as patient positioning on the treatment couch. Patient pelvic tilt and roll may change the relative relationship between the fiducial markers when projected onto the FPD during the acquisition of the radiograph. This possible pelvic rotation was simulated using the phantom and it was found that a 2 degree tilt or roll had a minor impact on the quality of registration. This result suggests that the $2 \mathrm{D}$ registration approach in the clinical scenario will be relatively insensitive to minor patient setup variations. 


\section{Catheter Path Comparison - "Pre-Treatment Imaging"}

Clinical based evaluation of prostate catheter implant movement can be performed by determining the shift in the catheter tips relative to implanted fiducial markers ${ }^{15-17}$. Our approach also measures the change of catheter position relative to the fiducial markers as these are used as the reference between the TPS and FPD space. This approach evaluates the geometric change of the implant that can possibly occur between two time points. More commonly this analysis is performed between treatment fractions ${ }^{18-21}$, and less often from treatment planning to prior to treatment delivery, where catheter shifts of -2.9 to $23.9 \mathrm{~mm}$ have been observed in the 1 to 3 hours interval between CT imaging and irradiation ${ }^{22}$. In the case of ultrasound based planning ${ }^{23}$ the time interval and possible catheter displacement may be less. The method of pre-treatment imaging presented in this work facilitates a robust approach to the evaluation of catheter implant movement.

The excellent agreement between planned and measured catheter tip positions for all catheters, having a maximum absolute deviation of $1.1 \mathrm{~mm}$, establishes the resolution of superior-inferior catheter movement detection. The comparison of all points along the catheter path is summarised in table 2, where the mean deviation observed is $0.7 \mathrm{~mm}$ or better for all six catheters.

The identification of the tip of the catheter in the TPS is determined by appending the centre of the CT marker inserted into the catheter during the CT scan. The uncertainty associated with this tip position arises from partial volume artefacts that occur across adjacent CT slices. We therefore express the uncertainty of the marker position as half the CT slice thickness (2.5 $\mathrm{mm}$ ), which in this case is $\pm 1.25 \mathrm{~mm}$. In Table 1 we observe a greater discrepancy for catheters 11 and 13, although still within this uncertainty, representing a larger influence of partial volume artefact. To improve the confidence in determining the catheter tip position in the treatment plan, it may be appropriate to scan across the catheter tip positions at a finer CT slice resolution. The appended marker tip position to which the planned (TPS) tip position is compared, is identified in the radiograph by determining the centre of the $10 \mathrm{~mm}$ spaced x-ray dwell position marker. The uncertainty in selecting exactly the centre pixel of the x-ray marker, which is defined to be the catheter tip position, is $\pm 0.8 \mathrm{~mm}$ ( 1 pixel).

This 2D evaluation of catheter movement between planning and treatment, can identify changes in the implant geometry that may be clinically significant. It has been reported in the clinic that implanted catheters move relative to the prostate primarily in the inferior direction 
(out of the patient) ${ }^{16,18}$, and in the prostatic radial direction. The observed radial changes in the catheter positions due to prostate volume changes, can be reported by $3 \mathrm{D}$ based pre-treatment imaging $^{24,25}$, but are small in magnitude and are less likely to impact the patients treatment. The long axis movement of the catheter is clinically more important to identify and account for ${ }^{24}$. A pre-treatment imaging approach such as this, will allow clinicians to verify catheter positions (implant stability) relative to the planned (TPS) positions and take corrective action if required.

\section{Dwell Position Comparison - "Source Tracking"}

The transformed planned dwell positions showed excellent agreement with the catheter paths visible in the radiograph (figure 6(c)). The measured dwell positions agreed with the planned dwell positions for all of the delivered dwells to within $1.4 \mathrm{~mm}$. The agreement of dwell positions is within the physical dimensions of the source which has a length of $3.6 \mathrm{~mm}$ and a specified position tolerance of $1.0 \mathrm{~mm}^{26}$.

The agreement between the measured dwell positions and the source position determined by the image subtraction method agreed on average by $0.8 \mathrm{~mm}$. This level of agreement is on the order of the pixel size of the imager and therefore it can be concluded that the two methods agree within the uncertainty of the detection system.

One limitation of this method is the 2D nature of the comparison of planned and measured positions. Although some situations can be contrived where the system may have trouble distinguishing between catheter positions (where two catheters are aligned one above the other and project on to the detector as one), this is unlikely in the prostate brachytherapy clinical situation. This 2D approach establishes a method for use in the clinic, enabling uptake of a system that can provide valuable information about the treatment, where at present options for systematic treatment verification are extremely limited.

The approach relies on the patient having implanted fiducial markers in the prostate allowing a relationship between the treatment plan and measurement space to be established. In the case 400 where fiducial markers are not present, a less powerful method of verification could be performed by registering the implanted catheters. This would achieve verification of the plan relative to the implant, but not necessarily inform the user of the relationship to the patient's anatomy. 
A proposed extension of this system will implement an error trapping algorithm based on data from these measured dwell positions. Consequently, during the treatment delivery, a real-time evaluation of plan to treatment agreement could be performed. This could enable an interrupt system to halt treatment while observed deviations are evaluated.

\section{Conclusion}

410 In this work we have demonstrated a method for monitoring a HDR brachytherapy treatment as it is delivered, and directly comparing the delivered treatment with the treatment plan in a system that can be easily implemented in the clinical workspace. The system uses a flat panel detector for both imaging and source tracking. Pre-treatment imaging enables visualisation of the implant before treatment delivery and identification of possible catheter displacement. In

415 this phantom study, we have shown that catheter tip positions can be measured to within 0.4 $\mathrm{mm}$ of the planned tip positions. Geometric changes in the implant can also be detected. Treatment delivery verification was performed by measuring source dwell positions and confirming that they agreed with the planned dwell positions on average within $0.6 \mathrm{~mm}$. This approach, using a flat panel detector for both imaging and source tracking, provides a noninvasive method of acquiring extensive information for verification in HDR prostate brachytherapy.

\section{Acknowledgements}

This research received funding from the Radiation Oncology Section of the Australian Priority-driven Collaborative Cancer Research Scheme.

\section{References}

${ }^{1}$ K. Tanderup, S. Beddar, C.E. Andersen, G. Kertzscher and J.E. Cygler, "In vivo dosimetry in brachytherapy," Med Phys 40, 070902 (2013).

${ }^{2}$ G. Kertzscher, A. Rosenfeld, S. Beddar, K. Tanderup and J.E. Cygler, "In vivo dosimetry: trends and prospects for brachytherapy," Br J Radiol 87, 20140206 (2014).

${ }^{3}$ B. Mijnheer, S. Beddar, J. Izewska and C. Reft, "In vivo dosimetry in external beam radiotherapy," Med Phys 40, 070903 (2013).

435 4 L.N. McDermott, M. Wendling, J.J. Sonke, M. van Herk and B.J. Mijnheer, "Replacing pretreatment verification with in vivo EPID dosimetry for prostate IMRT," Int J Radiat Oncol Biol Phys 67, 1568-1577 (2007). 
${ }^{5}$ J. Valentin, "Prevention of high-dose-rate brachytherapy accidents. ICRP Publication 97," Ann ICRP 35, 1-51 (2005).

$440{ }^{6} \mathrm{G}$. Kertzscher, C.E. Andersen and K. Tanderup, "Adaptive error detection for HDR/PDR brachytherapy: guidance for decision making during real-time in vivo point dosimetry," Med Phys 41, 052102 (2014).

${ }^{7}$ E.L. Seymour, S.J. Downes, G.B. Fogarty, M.A. Izard and P. Metcalfe, "In vivo real-time dosimetric verification in high dose rate prostate brachytherapy," Med Phys 38, 4785-4794 (2011).

$445{ }^{8} \mathrm{~A}$. Espinoza, et al., "The feasibility study and characterization of a two-dimensional diode array in "magic phantom" for high dose rate brachytherapy quality assurance," Med Phys 40, 111702 (2013).

${ }^{9} \mathrm{P}$. Jursinic, "Quality assurance measurements for high-dose-rate brachytherapy without film," J Appl Clin Med Phys 15, 4586 (2014).

$450{ }^{10}$ Y. Miyahara, H. Kitagaki, T. Nishimura, K. Itakura, S. Takahashi, M. Yokokawa, N. Uchida and T. Inomata, "Usefulness of direct-conversion flat-panel detector system as a quality assurance tool for high-dose-rate 192Ir source," J Appl Clin Med Phys 16, 5068 (2015).

${ }^{11}$ R.L. Smith, M.L. Taylor, L.N. McDermott, A. Haworth, J.L. Millar and R.D. Franich, "Source position verification and dosimetry in HDR brachytherapy using an EPID," Med Phys 40, 111706 (2013).

${ }^{12}$ J.M. Balter, K.L. Lam, H.M. Sandler, J.F. Littles, R.L. Bree and R.K. Ten Haken, "Automated localization of the prostate at the time of treatment using implanted radiopaque markers: technical feasibility," Int J Radiat Oncol Biol Phys 33, 1281-1286 (1995).

${ }^{13}$ K.S. Arun, T.S. Huang and S.D. Blostein, "Least-squares fitting of two 3-d point sets," IEEE transactions on pattern analysis and machine intelligence 9, 698-700 (1987).

${ }^{14}$ Varian (2000). Portal Vision aS500 Reference Manual. P.A. Varian Medical Systems, CA, USA.

${ }^{15}$ R. Holly, G.C. Morton, R. Sankreacha, N. Law, T. Cisecki, D.A. Loblaw and H.T. Chung, "Use of cone-beam imaging to correct for catheter displacement in high dose-rate prostate brachytherapy," Brachytherapy 10, 299-305 (2011).

$465{ }^{16}$ Y. Kim, I.C. Hsu and J. Pouliot, "Measurement of craniocaudal catheter displacement between fractions in computed tomography-based high dose rate brachytherapy of prostate cancer," $\mathbf{J}$ Appl Clin Med Phys 8, 2415 (2007).

${ }^{17}$ C.D. Fox, T. Kron, M. Leahy, G. Duchesne, S. Williams, K.H. Tai, A. Haworth, A. Herschtal and F. Foroudi, "Interfraction patient motion and implant displacement in prostate high dose rate brachytherapy," Med Phys 38, 5838-5843 (2011).

${ }^{18}$ P.J. Hoskin, P.J. Bownes, P. Ostler, K. Walker and L. Bryant, "High dose rate afterloading brachytherapy for prostate cancer: catheter and gland movement between fractions," Radiother Oncol 68, 285-288 (2003).

475 brachytherapy in the treatment of prostate cancer," Int J Radiat Oncol Biol Phys 46, 1205-1211 (2000).

${ }^{20}$ T. Simnor, S. Li, G. Lowe, P. Ostler, L. Bryant, C. Chapman, D. Inchley and P.J. Hoskin, "Justification for inter-fraction correction of catheter movement in fractionated high dose-rate brachytherapy treatment of prostate cancer," Radiother Oncol 93, 253-258 (2009).

$480{ }^{21}$ S. Kawakami, et al., "Catheter displacement prior to the delivery of high-dose-rate brachytherapy in the treatment of prostate cancer patients," Journal of contemporary brachytherapy 6, 161-166 (2014).

${ }^{22}$ M. Whitaker, G. Hruby, A. Lovett and N. Patanjali, "Prostate HDR brachytherapy catheter displacement between planning and treatment delivery," Radiother Oncol 101, 490-494 (2011).

$485{ }^{23}$ D. Batchelar, M. Gaztanaga, M. Schmid, C. Araujo, F. Bachand and J. Crook, "Validation study of ultrasound-based high-dose-rate prostate brachytherapy planning compared with CT-based planning," Brachytherapy 13, 75-79 (2014).

${ }^{24}$ W. Foster, J.A. Cunha, I.C. Hsu, V. Weinberg, D. Krishnamurthy and J. Pouliot, "Dosimetric impact of interfraction catheter movement in high-dose rate prostate brachytherapy," Int J Radiat Oncol Biol Phys 80, 85-90 (2011). 
${ }^{25}$ Y. Huang, B. Miller, A. Doemer, D. Babij, S. Kumar, R. Frontera, T. Nurushev, I.J. Chetty and I. Aref, "Online correction of catheter movement using CT in high-dose-rate prostate brachytherapy," Brachytherapy 12, 260-266 (2013).

${ }^{26}$ Nucletron, "microSelectron V3 Reference Manual," 190.015ENG-03, Nucletron an Elekta Company, Stockholm, Sweeden. 


\section{University Library}

\section{- M M N E R VA A gateway to Melbourne's research publications}

Minerva Access is the Institutional Repository of The University of Melbourne

Author/s:

Smith, RL;Haworth, A;Panettieri, V;Millar, JL;Franich, RD

Title:

A method for verification of treatment delivery in HDR prostate brachytherapy using a flat panel detector for both imaging and source tracking

Date:

2016-05-01

Citation:

Smith, R. L., Haworth, A., Panettieri, V., Millar, J. L. \& Franich, R. D. (2016). A method for verification of treatment delivery in HDR prostate brachytherapy using a flat panel detector for both imaging and source tracking. MEDICAL PHYSICS, 43 (5), pp.2435-2442. https:// doi.org/10.1118/1.4946820.

Persistent Link:

http://hdl.handle.net/11343/291232 